\title{
A competitive location model to obtain a specific market share while ranking facilities by shorter travel time
}

\author{
Naeme Zarrinpoor and Mehdi Seifbarghy \\ Technical and Engineering Department, Alzahra University, Deh Vanak, Tehran, Iran
}

\begin{abstract}
Competitive location deals with the problem of locating new facilities to provide a service (or goods) to the customers of a given geographical area where other facilities (competitors) offering the same service are already present. The new facilities will have to compete with the existing facilities for capturing the market share. This paper proposes a new model in which a new entering firm desires to obtain a specific percentage of the market share in such a way as to minimize the total costs. The total costs consist of costs earmarked for installing the new located facilities and traveling as well as the waiting costs of the customers who receive service from the facilities. Two heuristics based on genetic algorithm and tabu search are proposed to solve the model. A number of numerical examples are designed and solved in order to assess the performance of the proposed heuristics.
\end{abstract}

Keywords: Competitive location, Queuing, Congested systems, Market capture. 